DOI: https://doi.org/10.46296/yc.v4i7.0055

\title{
CRUCITA, DESTINO TURÍSTICO: REALIDAD EMPRESARIAL Y DESAFÍOS PRODUCTIVOS POST 16A
}

\section{CRUCITA, TOURIST DESTINATION: BUSINESS REALITY AND POST 16A PRODUCTIVE CHALLENGES}

\author{
Feijó-Cuenca Tito Eliecer ${ }^{1 *}$; Feijó-Cuenca Nilba Priscila ${ }^{2}$; Quiroz-Peñarrieta \\ Irlanda Isabel ${ }^{3}$ \\ 1, 2 Universidad Técnica de Manabí, UTM. Portoviejo, Ecuador. \\ ${ }^{3}$ Contador público autorizado. Portoviejo, Ecuador.
}

*Correo: tito.feijo@utm.edu.ec

\begin{abstract}
Resumen
El presente trabajo de investigación tuvo como propósito general analizar a la parroquia Crucita como destino turístico y sus perspectivas de crecimiento empresarial del cantón Portoviejo luego del terremoto del 16A. A través de metodología de carácter no experimental, cualitativa y cuantitativa, se analizó información relacionada con catástrofes naturales en destinos turísticos, así como información relevante de Crucita obtenida de la base de datos del Ministerio de Turismo y del Servicio de Rentas Internas. Los resultados de la investigación permitieron obtener una visión general de los negocios según la actividad productiva, que inciden en el establecimiento de líneas de intervención para el desarrollo de proyectos que contribuyan al fortalecimiento y progreso de Crucita como destino turístico.
\end{abstract}

Palabras claves: Catástrofe natural, sector turístico, creación de negocios, actividad productiva.

\begin{abstract}
The general purpose of this research work was to analyze the Crucita parish as a tourist destination and its prospects for business growth in the Portoviejo canton after the $16 \mathrm{~A}$ earthquake. Through a non-experimental, qualitative and quantitative methodology, information related to natural disasters in tourist destinations was analyzed, as well as relevant information on Crucita obtained from the database of the Ministry of Tourism and the Internal Revenue Service. The results of the research allowed to obtain an overview of the businesses according to the productive activity, which affect the establishment of lines of intervention for the development of projects that contribute to the strengthening and progress of Crucita as a tourist destination.
\end{abstract}

Keywords: Natural catastrophe, tourism sector, business creation, productive activity.

Información del manuscrito:

Fecha de recepción: 13 de mayo de 2020.

Fecha de aceptación: 09 de julio de 2020.

Fecha de publicación: 10 de julio de 2020. 


\section{Introducción}

Fueron setenta y cinco segundos que remecieron gran parte del Ecuador en Manabí y Esmeraldas, el 16 de abril de 2016. El Sistema Económico Latinoamericano y del Caribe (2016), manifestó que industrias como el comercio, la agricultura y la pesca, la manufactura y turismo, fueron muy afectadas y su infraestructura productiva se vio devastada, parcialmente destruidas 0 mermadas. Con ello vino no solo un decrecimiento en la economía, que la SENPLADES estima en un $0,7 \%$, sino un efecto en el empleo, que ya venía siendo golpeado producto de la crisis del país.

De acuerdo con datos proporcionados por el Comité para la Reconstrucción y Reactivación Productiva (2016), este trágico acontecimiento dejó 661 personas fallecidas, 12 desaparecidos, 4859 atenciones a heridos y $80 \mathrm{mil}$ desplazados por pérdida o daño de sus viviendas. Se estima que $500 \mathrm{mil}$ personas quedaron con limitación en inseguridad alimentaria, $350 \mathrm{mil}$ personas con acceso restringido a agua en los primeros días luego del terremoto, 170 mil personas con afectaciones en sus medios de vida y 200 mil personas con afectaciones psicológicas.

Aún en países como Ecuador poseedores de una sorprendente naturaleza con una elevada biodiversidad, diversidad cultural, con potencial turístico de primer orden en aventura, arte, cultura y naturaleza (García Casado, M., \& Palacios Estremera, M., 2003); las cifras evidencian de manera tangible que los desastres naturales traen consigo una desaceleración de grandes proporciones en la economía.

El movimiento sísmico acontecido en Ecuador el 16 de abril de 2016, afectó a la parroquia Crucita conocida turísticamente como "Crucita la Bella", principal balneario del cantón Portoviejo, localizada en la costa de la provincia de Manabí, la cual cuenta con un área abierta de $13 \mathrm{~km}$ de hermosas playas, con una aceptable planta de establecimientos que ofrecen servicios de alojamientos, comidas, bebidas y diversión. Desde hace muchos años la población de Crucita se dedica principalmente a el turismo, pero no es el único sector que genera fuentes de ingresos para 
su población, destacándose como sus principales actividades productivas la pesca artesanal, la agricultura y el turismo.

El presente artículo tuvo como propósito general analizar a la parroquia Crucita como destino turístico y sus perspectivas de crecimiento empresarial del cantón Portoviejo luego del terremoto del 16A. La metodología utilizada fue de carácter cuantitativa, debido a que esta se usa principalmente para comparar datos con orientación numérica, y el rigor científico se fundamenta en la fiabilidad y la validez de los datos. Para este trabajo se utilizaron bases de datos oficiales del Ministerio de Turismo y del Servicio de Rentas Internas, realizando un seguimiento al crecimiento y decrecimiento de diferentes sectores productivos de Crucita durante el año del acontecimiento y tres años posteriores a dicho acontecimiento.

Como resultado se pusieron en evidencia las dificultades económicas que sufren la mayoría de los sectores productivos en Crucita; además, se demostró el crecimiento y decrecimiento de ciertos fragmentos que contribuyen al desarrollo sustentable y sostenible de Crucita.

\subsection{Catástrofe natural}

En el desarrollo de la teoría de las catástrofes es posible realizar una primera y fundamental distinción entre los dos términos: "desastre", que originariamente significa "mala estrella", haciendo referencia a un evento perjudicial, cuyas consecuencias pueden ser absorbidas por un sistema, en tiempos relativamente breves. Por su parte, "catástrofe" identifica una crisis con efectos de "alteración" materiales, organizativos, sociales, irreversibles para todo el sistema (Placanica, 1991).

Los sucesos incluidos en la categoría "desastres", es una condición vaga que agrupa fenómenos muy diferentes entre sí, ya sea por sus dimensiones o por sus particularidades, como: terremotos, erupciones volcánicas, epidemias, guerras, deslizamientos de tierra, inundaciones, sequías, accidentes tecnológicos y nucleares. Los dos términos usados con mayor frecuencia en la literatura específica son 'desastre' y 'catástrofe', con 
sinónimos como 'cataclismo' y 'calamidad', los cuales son menos utilizados (Da Cruz et ál., 2003).

El origen de las catástrofes naturales se da por la liberación de energía de elementos naturales tales como el agua, fuego, tierra, aire, siendo estas perjudiciales en la medida que afecten a la colectividad humana: Terremotos, erupciones volcánicas, tsunamis, huracanes, tifones, maremotos, tempestades de nieve (Villalibre, 2013).

En Ecuador específicamente en la provincia de Manabí, el 16 de abril del año 2016 aconteció un fuerte terremoto de 7,8 de magnitud en la escala de Richter, esta catástrofe natural trajo consigo un total de 661 personas fallecidas, convirtiéndose en el más mortal de Sudamérica desde el de Colombia en 1999, que mató a más de 1.000 personas (Velásquez, 2017).

Este acontecimiento es considerado una de las catástrofes naturales más graves por las que el Ecuador ha pasado en los últimos 70 años, el mismo afectó a la costa norte del país, en gran parte los hoteles, calles e infraestructura quedaron destrozadas. Tanto Pedernales y algunas zonas de Portoviejo y Manta quedaron prácticamente irreconocibles; muchas de estas zonas son turísticas por excelencia y hacen parte de los más de 1600 kilómetros de playas del clima del Ecuador (Velásquez, 2017).

Tabla 1. Reporte de personas fallecidas y desaparecidas en Ecuador durante el $16 \mathrm{~A}$

\begin{tabular}{|c|c|c|c|}
\hline $\begin{array}{l}\text { PROVIN } \\
\text { CIA }\end{array}$ & CANTÓN & $\begin{array}{l}\text { PERSO } \\
\text { NAS } \\
\text { FALLE } \\
\text { CIDAS }\end{array}$ & $\begin{array}{c}\text { PERSONA } \\
\text { S } \\
\text { DESAPAR } \\
\text { ECIDAS }\end{array}$ \\
\hline \multirow{2}{*}{$\begin{array}{l}\text { ESMER } \\
\text { ALDAS }\end{array}$} & MUISNE & & 1 \\
\hline & SUBTOTAL & & 1 \\
\hline \multirow{15}{*}{ MANABÍ } & PORTOVIEJO & 133 & 4 \\
\hline & MANTA & 211 & 1 \\
\hline & PEDERNALES & 173 & 2 \\
\hline & SAN VICENTE & 38 & \\
\hline & CHONE & 6 & \\
\hline & BOLIVAR & 8 & \\
\hline & EL CARMEN & 8 & \\
\hline & JAMA & 27 & \\
\hline & SUCRE & 28 & \\
\hline & ROCAFUERTE & 8 & \\
\hline & FLAVIO ALFARO & 6 & \\
\hline & PUERTO LÓPEZ & & 1 \\
\hline & TOSAGUA & 1 & \\
\hline & $\begin{array}{l}\text { EN ZONA NO } \\
\text { DETERMINADA }\end{array}$ & & 2 \\
\hline & SUBTOTAL & 646 & 10 \\
\hline \multirow{3}{*}{$\begin{array}{c}\text { SANTO } \\
\text { DOMIN } \\
\text { GO }\end{array}$} & $\begin{array}{l}\text { SANTO } \\
\text { DOMINGO }\end{array}$ & 4 & \\
\hline & LA CONCORDIA & 1 & \\
\hline & SUBTOTAL & 5 & \\
\hline \multirow{4}{*}{$\begin{array}{c}\text { GUAYA } \\
\text { S }\end{array}$} & GUAYAQUIL & 3 & \\
\hline & DAULE & 2 & \\
\hline & $\begin{array}{l}\text { SAMBORONDÓ } \\
\mathrm{N}\end{array}$ & 2 & \\
\hline & SUBTOTAL & 7 & \\
\hline \multirow{2}{*}{$\begin{array}{l}\text { PICHIN } \\
\text { CHA }\end{array}$} & QUITO & 1 & \\
\hline & SUBTOTAL & 1 & \\
\hline \multirow{2}{*}{$\begin{array}{c}\text { CHIMBO } \\
\text { RAZO }\end{array}$} & COLTA & 1 & \\
\hline & SUBTOTAL & 1 & \\
\hline \multirow{3}{*}{ NAPO } & TENA & & 1 \\
\hline & SUBTOTAL & 0 & 1 \\
\hline & TOTAL & 661 & 12 \\
\hline
\end{tabular}

Fuente: Naciones unidas para la coordinación de asuntos Humanitarios, 2016.

Elaborado por: Investigadores.

Las zonas con mayor afectación fueron las provincias de Manabí, Esmeraldas, Santa Elena, Guayas, 
Santo Domingo y Los Ríos, mismas que fueron declaradas por el Gobierno como en "estado de emergencia". La provincia más golpeada fue Manabí; uno de sus cantones, Pedernales $\quad(55.000$ habitantes), fue declarado como "zona de desastre" (Naciones Unidas para la Coordinación de Asuntos Humanitarios, 2016)

Tabla 2. Afectación por daños eléctricos.

\begin{tabular}{|l|c|}
\hline \multicolumn{1}{|c|}{ PROVINCIA } & $\begin{array}{c}\text { CON SERVICIO } \\
\text { ELÉCTRICO }\end{array}$ \\
\hline ESMERALDAS & $89 \%$ \\
\hline MANABÍ & $0 \%$ \\
\hline LOS RIIOS & $57 \%$ \\
\hline SANTO DOMINGO & $92 \%$ \\
\hline GUAYAS & $98 \%$ \\
\hline SANTA ELENA & $98 \%$ \\
\hline
\end{tabular}

Fuente: CENACE, 2016.

Elaborado por: Investigadores.

Tras el terremoto del 16 de abril del 2016, varias provincias se vieron afectadas en la infraestructura de distribución del sistema eléctrico, siendo en la provincia de Manabí donde mayores daños se reportaron.

Figura 1. Pérdida de empleo tras el terremoto del 16 de abril 2016.

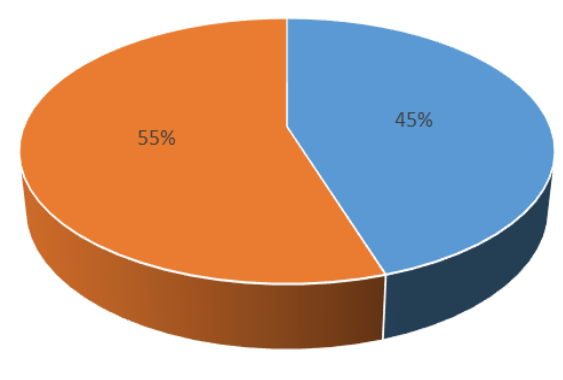

- pérdidas de empleos informales "pérdidas de empleos formales

Fuente: Secretaria Nacional de Planificación y Desarrollo (SENPLADES), 2016.

Elaborado por: Investigadores.
La Secretaria Nacional de Planificación y Desarrollo (SENPLADES), realizó la cuantificación de las afectaciones causadas por el terremoto en relación a la pérdida de empleo, en dicho estudio se estimó una pérdida de 21.823 puestos de trabajo, el $45 \%$ formales y el $55 \%$ informales, de estos el $97 \%$ corresponde al sector productivo, el $47 \%$ al sector de la agricultura, acuacultura pesca, el $34 \%$ al sector comercio, $9 \%$ a los servicios turísticos, el $7 \%$ a la industria manufacturera $y, \quad$ el $3 \%$ restante a los sectores sociales y de infraestructura.

\subsection{Sector turístico}

La Organización Mundial del Turismo (OMT, 1999), describe el turismo como las actividades que realizan las personas durante sus viajes y estancias en lugares distintos al de su entorno habitual. Igualmente, los fundadores de la escuela humanística definieron el turismo como un conjunto de relaciones y fenómenos producidos por el desplazamiento y la permanencia temporal de personas fuera de su lugar de residencia habitual, sin que sean motivados por 
el lucro (Hunziker y Krapf, 1942:5). Mientras que Cuervo (1967), manifiesta que el turismo es un conjunto bien definido de relaciones, servicios e instalaciones que se generan en virtud de ciertos desplazamientos humanos.

En términos económicos el turismo se ha convertido en uno de los ejes de desarrollo más importantes para el Ecuador, siendo este un sector muy dinámico y con una gran capacidad de desarrollo, se encuentra ligado al cambio de la matriz productiva del país siendo fuente de ingresos de muchas familias que dedican su vida a emprender turísticamente (Rivadeneira, 2014), es así que el turismo conlleva una serie de actividades y acciones que realizan las personas en beneficio de su entorno y de sus visitantes (Moragues, 2006).

Tabla 3. Registros Turísticos de Ecuador por provincias.

\begin{tabular}{|c|c|c|c|}
\cline { 2 - 4 } \multicolumn{1}{c|}{} & PROVINCIA & $\begin{array}{c}\text { No DE } \\
\text { REGISTRO } \\
\text { S }\end{array}$ & $\begin{array}{c}\text { Porcent } \\
\text { aje }\end{array}$ \\
\hline 1 & GUAYAS & 5803 & $24 \%$ \\
\hline 2 & PICHINCHA & 5515 & $23 \%$ \\
\hline 3 & AZUAY & 2332 & $10 \%$ \\
\hline 4 & MANABI & 1625 & $7 \%$ \\
\hline 5 & TUNGURAHUA & 1322 & $5 \%$ \\
\hline 6 & SANTA ELENA & 918 & $4 \%$ \\
\hline 7 & GALÁPAGOS & 884 & $4 \%$ \\
\hline 8 & EL ORO & 842 & $3 \%$ \\
\hline 9 & LOJA & 579 & $2 \%$ \\
\hline 10 & IMBABURA & 576 & $2 \%$ \\
\hline
\end{tabular}

\begin{tabular}{|c|l|c|c|}
11 & CHIMBORAZO & 550 & $2 \%$ \\
\hline 12 & COTOPAXI & 398 & $2 \%$ \\
\hline 13 & ESMERALDAS & 397 & $2 \%$ \\
\hline 14 & NAPO & 368 & $2 \%$ \\
\hline 15 & LOS RÍOS & 355 & $1 \%$ \\
\hline 16 & CAÑAR & 338 & $1 \%$ \\
\hline \multirow{2}{*}{17} & $\begin{array}{l}\text { SANTO } \\
\text { DOMINGO DE } \\
\text { LOS } \\
\text { TSÁCHILAS }\end{array}$ & 338 & $1 \%$ \\
\hline 18 & $\begin{array}{l}\text { MORONA } \\
\text { SANTIAGO }\end{array}$ & 293 & $1 \%$ \\
\hline 19 & PASTAZA & 252 & $1 \%$ \\
\hline 20 & SUCUMBIOS & 215 & $1 \%$ \\
\hline 21 & ORELLANA & 199 & $1 \%$ \\
\hline 22 & BOLIVAR & 165 & $1 \%$ \\
\hline 23 & $\begin{array}{l}\text { ZAMORA } \\
\text { CHINCHIPE }\end{array}$ & 115 & $0 \%$ \\
\hline 24 & CARCHI & 77 & $0 \%$ \\
\hline & Total general & $\mathbf{2 4 4 5 6}$ & $\mathbf{1 0 0} \%$ \\
\cline { 2 - 4 } & & \\
\cline { 3 - 4 } & & & \\
\hline
\end{tabular}

Fuente: Ministerio de turismo del Ecuador 2019.

Elaborado por: Investigadores.

Según el Ministerio de Turismo del Ecuador, Manabí se ubica en un cuarto lugar con 1625 registros turísticos, hay que destacar que esto en gran parte se debe al clima de Manabí que oscila entre subtropical seco a tropical húmedo y tropical extremadamente húmedo, aunque la temperatura no es uniforme en toda la provincia, la temperatura media en Portoviejo, la capital, es de 25 grados centígrados y en la ciudad de Manta, es de 23,8 grados centígrados. Además, la gastronomía manabita es reconocida a nivel nacional e internacional por su originalidad y exquisitez, sus platos típicos constituyen un verdadero atractivo para propios extraños. Según Trejo, 
D. (2013) indica que Manabí cuenta con atractivos naturales, culturales y paisajísticos, entre las principales playas encontramos El Murciélago y Tarqui en Manta; Crucita en Portoviejo; también, San Jacinto, San Clemente, San Vicente, Boca de Briceño, Canoa, Pedernales, Cojimíes, Puerto Cayo, Puerto López y el Parque Nacional Machalilla.

Tabla 4. Registros Turísticos de Manabí por cantones.

\begin{tabular}{|c|c|c|c|}
\hline & CANTÓN & $\begin{array}{c}\text { № DE } \\
\text { REGISTROS }\end{array}$ & \\
\hline 1 & MANTA & 611 & $37,60 \%$ \\
\hline 2 & PORTOVIEJO & 320 & $19,69 \%$ \\
\hline 3 & $\begin{array}{l}\text { PUERTO } \\
\text { LÓPEZ }\end{array}$ & 175 & $10,77 \%$ \\
\hline 4 & PEDERNALES & 116 & $7,14 \%$ \\
\hline 5 & SUCRE & 105 & $6,46 \%$ \\
\hline 6 & SAN VICENTE & 74 & $4,55 \%$ \\
\hline 7 & JIPIJAPA & 64 & $3,94 \%$ \\
\hline 8 & EL CARMEN & 25 & $1,54 \%$ \\
\hline 9 & CHONE & 23 & $1,42 \%$ \\
\hline 10 & MONTECRISTI & 22 & $1,35 \%$ \\
\hline 11 & BOLÍVAR & 17 & $1,05 \%$ \\
\hline 12 & JAMA & 16 & $0,98 \%$ \\
\hline 13 & SANTA ANA & 15 & $0,92 \%$ \\
\hline 14 & ROCAFUERTE & 11 & $0,68 \%$ \\
\hline 15 & TOSAGUA & 7 & $0,43 \%$ \\
\hline 16 & JARAMIJO & 6 & $0,37 \%$ \\
\hline 17 & PAJÁN & 6 & $0,37 \%$ \\
\hline 18 & $\begin{array}{l}\text { FLAVIO } \\
\text { ALFARO }\end{array}$ & 5 & $0,31 \%$ \\
\hline 19 & JUNIN & 5 & $0,31 \%$ \\
\hline 20 & 24 DE MAYO & 1 & $0,06 \%$ \\
\hline 21 & PICHINCHA & 1 & $0,06 \%$ \\
\hline & Total general & 1625 & $100 \%$ \\
\hline
\end{tabular}

Fuente: Ministerio de turismo del Ecuador 2019.

Elaborado por: Investigadores.

Manabí es tierra de encanto, llena de pasión y belleza, conjugada con la amabilidad de su gente, lo que la convierten en un imán para turistas nacionales y extranjeros. Uno de sus cantones, Manta, es la que mayor planta turística posee (611) y es visitada anualmente por importantes cruceros, seguida de Portoviejo (320) siendo esta la capital de los manabitas y el centro de comercio de la provincia.

Manabí se caracteriza por sus pintorescos pueblos de pescadores, es una zona privilegiada por sus 350 kilómetros de playa, además de montañas cascadas y ríos. La tradición y cultura de su gente se puede apreciar en una variada muestra de artesanías en mimbre, cerámica, sombreros de paja, cestería. Cuenta con 4 rutas turísticas que son: Ruta del Cacao, ruta del Café, ruta de la Paja Toquilla y la ruta del Colibrí.

Tabla 5. Registros Turísticos de Portoviejo por parroquias.

\begin{tabular}{|c|c|c|c|}
\hline & PARROQUIA & $\begin{array}{c}\text { № DE } \\
\text { REGISTROS }\end{array}$ & \\
\hline 1 & $\begin{array}{l}\text { PORTOVIEJO } \\
\text { (CAB. } \\
\text { CANTONAL Y } \\
\text { CAPITAL DE } \\
\text { PROVINCIA) }\end{array}$ & 226 & $70,63 \%$ \\
\hline 2 & CRUCITA & 50 & $15,63 \%$ \\
\hline 3 & PORTOVIEJO & 11 & $3,44 \%$ \\
\hline 4 & $\begin{array}{l}18 \mathrm{DE} \\
\text { OCTUBRE }\end{array}$ & 9 & $2,81 \%$ \\
\hline 5 & 12 DE MARZO & 7 & $2,19 \%$ \\
\hline 6 & $\begin{array}{l}\text { ANDRÉS DE } \\
\text { VERA }\end{array}$ & 4 & $1,25 \%$ \\
\hline 7 & $\begin{array}{l}\text { FRANCISCO } \\
\text { PACHECO }\end{array}$ & 2 & $0,63 \%$ \\
\hline 8 & PICOAZÁ & 2 & $0,63 \%$ \\
\hline 9 & $\begin{array}{l}\text { RIOCHICO (RIO } \\
\text { CHICO) }\end{array}$ & 2 & $0,63 \%$ \\
\hline
\end{tabular}




\begin{tabular}{|c|l|c|c|}
10 & SAN PABLO & 2 & $0,63 \%$ \\
\hline 11 & $\begin{array}{l}\text { ABDÓN } \\
\text { CALDERÓN } \\
\text { (SAN } \\
\text { FRANCISCO) }\end{array}$ & 1 & $0,31 \%$ \\
\hline 12 & $\begin{array}{l}\text { ALHAJUELA } \\
\text { (BAJO } \\
\text { GRANDE) }\end{array}$ & 1 & $0,31 \%$ \\
\hline 13 & COLÓN & 1 & $0,31 \%$ \\
\hline 14 & $\begin{array}{l}\text { PORTOVIEJO, } \\
\text { CABECERA } \\
\text { CANTONAL Y } \\
\text { CAPITAL } \\
\text { PROVINCIAL }\end{array}$ & 1 & $0,31 \%$ \\
\hline 15 & SAN PLACIDO & 1 & $0,31 \%$ \\
\hline & Total general & $\mathbf{3 2 0}$ & $\mathbf{1 0 0} \%$ \\
\hline
\end{tabular}

Fuente: Ministerio de turismo del Ecuador 2019.

Elaborado por: Investigadores.

\section{Materiales y métodos}

En un primer momento, a partir publicaciones y bibliografía relacionada con catástrofes naturales en destinos turísticos, se logró fundamentar el estado del arte sujeto de nuestro estudio, con un análisis deductivo - inductivo, a través de la presentación de información a nivel país de las afectaciones sufridas tras el terremoto, para luego pasar a información detallada de la provincia, del cantón Portoviejo y finalmente de la parroquia objeto de observación que es Crucita.

En un segundo momento, se procedió al análisis minucioso de las bases de datos emitidas por organismos oficiales como el
Gobierno

Autónomo

Descentralizado de Portoviejo (siendo esta la más importantes a escala local), y las del Ministerio de Turismo, y Servicio de Rentas Internas (siendo estas las más importantes a escala nacional). Esto permitió a los investigadores hacer una comparación entre la información que cada organismo administra para poner en perspectiva el enfoque económico actual de este punto geográfico y las pretensiones de crecimiento turístico que la actual administración pública desea proporcionar a esta zona para impulsar su crecimiento productivo tras el terremoto.

La aplicación de metodología cualicuantitativa, permitió llegar a conclusiones puntuales a través de los resultados presentados en la gráfica comparativa de los datos obtenidos de diferentes instituciones públicas de seguimiento y control relevantes de la parroquia Crucita del cantón Portoviejo en la Provincia de Manabí, mismos que se presentan en los siguientes apartados. 


\section{Resultados}

\subsection{Parroquia Crucita}

Crucita, está ubicada en la provincia de Manabí, a 30 minutos de Portoviejo y 30 minutos de Manta, según Muñoz (2012) indica que esta hermosa playa es uno de los destinos turísticos preferidos del Ecuador, gracias a su agradable clima durante todos los días del año. Crucita constituye un escenario natural propio para la realización de importantes y exitosos eventos, que constantemente atraen hasta sus playas a turistas nacionales $y$ extranjeros, que la visitan para disfrutar de las bondades de su mar y sus hermosos paisajes.

Tabla 6. Análisis comparativo de datos Ministerio de Turismo vs. GAD Portoviejo.

\begin{tabular}{|c|c|c|c|c|}
\hline \multirow[b]{2}{*}{ Alimentos y Bebidas } & \multicolumn{2}{|c|}{ MINTUR } & \multicolumn{2}{|c|}{$\begin{array}{l}\text { GAD } \\
\text { PORTOVIEJO }\end{array}$} \\
\hline & 30 & $60 \%$ & 23 & $46 \%$ \\
\hline Fuente de soda & 1 & $2 \%$ & & $0 \%$ \\
\hline Restaurante & 29 & $58 \%$ & & $0 \%$ \\
\hline Alojamiento & 18 & $36 \%$ & 25 & $50 \%$ \\
\hline Hostal & 8 & $16 \%$ & 8 & $16 \%$ \\
\hline Hostería & 2 & $4 \%$ & 4 & $8 \%$ \\
\hline Hotel & 8 & $16 \%$ & 8 & $16 \%$ \\
\hline Cabañas & & & 2 & $4 \%$ \\
\hline Hospedaje & & & 1 & $2 \%$ \\
\hline Pensión & & & 1 & $2 \%$ \\
\hline Suite & & & 1 & $2 \%$ \\
\hline Centro de Turismo Comunitario & 1 & $2 \%$ & & $0 \%$ \\
\hline Centro de Turismo Comunitario & 1 & $2 \%$ & & $0 \%$ \\
\hline Operación e Intermediación & 1 & $2 \%$ & 1 & $2 \%$ \\
\hline Operador turístico & 1 & 1 & 1 & $2 \%$ \\
\hline Agencia de viajes & 1 & $2 \%$ & 1 & $2 \%$ \\
\hline Agencia de viajes & & & 1 & $2 \%$ \\
\hline Iotal general & 50 & $100 \%$ & 50 & $100 \%$ \\
\hline
\end{tabular}

Fuente: Ministerio de Turismo del Ecuador, 2019; GAD de Portoviejo, 2019.

Elaborado por: Investigadores.
En los alrededores de la playa de Crucita se pueden encontrar varios hoteles, restaurantes, fuentes de sodas en las que se puede disfrutar de momentos agradables junto amigos y familiares. Cabe señalar que de los turistas que visitan Crucita, el 95\% lo hace en el día, es decir, los turistas no pernoctan en el lugar, debido a la cercanía de ciudades como Portoviejo y Manta, y el $5 \%$ es abastecido por los hoteles que se encuentran en la parroquia.

Las bases de datos analizadas tanto del Ministerio de Turismo como del GAD de Portoviejo muestran información que no se corresponde, lo cual deja una gran brecha del entorno económico del destino turístico de Crucita.

\subsection{Creación de negocios}

El 16 de abril de 2016 se registró un movimiento telúrico con una magnitud de 7,8 grados en la escala de Ricther, el sismo tuvo una duración de 75 segundos, durante los siguientes días se registraron más de 300 réplicas entre 6,0 y 6,7 de magnitud en la escala de Richter (García, Doumet, Alfonso, 2018). Crucita fue una de las parroquias afectadas por tal 
acontecimiento, $y$ al igual que muchas otras tuvo que adaptarse hacia un desarrollo local, el cual radica según Formichella (2014) en el proceso dinámico e integrador en zonas o localidades, mejorando las condiciones de vida de los habitantes. Son las personas que se arriesgan a emprender quienes hacen negocios exitosos, desarrollando nuevas ideas o formas de enfocar el mercado.

Figura 2. Negocios aperturados por año en Crucita.

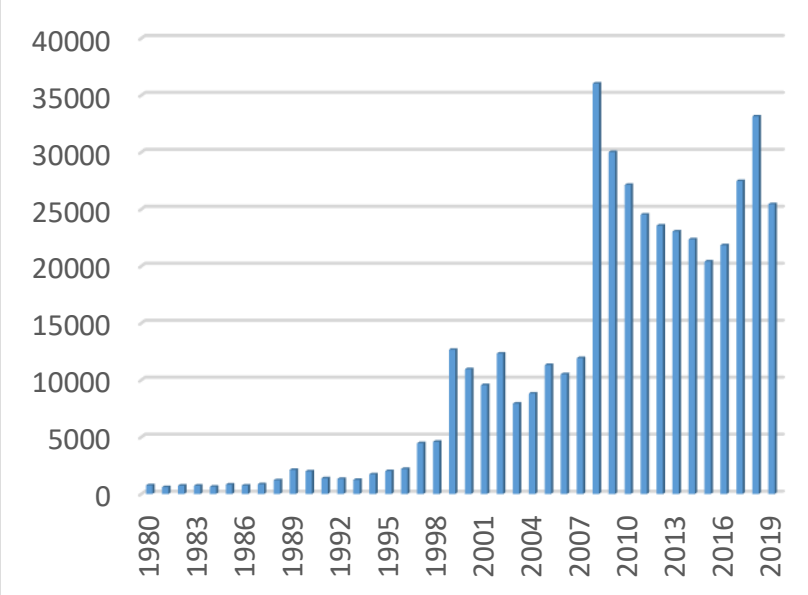

Fuente: Servicio de Rentas Internas del Ecuador (SRI).

Elaborado por: Investigadores.

Según la base de datos del SRI la población de Crucita muestra interés por incursionar en actividades productivas, esto se refleja en la apertura anual de nuevos emprendimientos, siendo los años 2008 y 2018 , los que muestran los puntos más altos en la apertura de nuevos negocios. Las estadísticas nos permiten observar que a pesar de la afectación sufrida durante el 2016, las iniciativas emprendedoras no cesaron, manteniéndose una curva ascendente hasta el 2018, misma que no se aprecia en su total magnitud al 2019, por poseer una data inconclusa del período al momento del corte.

\subsection{Actividad productiva}

El desarrollo del turismo en una región dinamiza toda la economía beneficiando a la comunidad. Este impacto, efecto derrame, se analiza a través de la producción y el consumo de los diferentes bienes y servicios que se generan como resultado de la demanda inducida por esta actividad (SANTAFETURISMO, 2017). Como lo manifestó Weyl (2014), Crucita es un pueblo pequeño, pero con un centro turístico maravilloso, promoviendo el turismo, beneficio económico de la comunidad. De ahí que el turismo es una actividad multisectorial y multidisciplinaria en la que participan diferentes áreas productivas como la agricultura, construcción, fabricación y de sectores públicos y privados para 
proporcionar los bienes y los servicios utilizados (Zilberstein, 2017).

De acuerdo con la data analizada las actividades en las que más iniciativa existe para la generación de negocios son la venta al por menor de prendas de vestir y peletería en establecimientos especializados, y cultivo de maíz; mostrando una faceta poco difundida en los informes que generalmente se muestran sobre las actividades económicas de esta parroquia, visualizando desde una mirada global el gran potencial del sector agropecuario para generar movimiento monetario.

Tabla 7. Actividades productivas aperturadas por año.

\begin{tabular}{|c|l|c|c|c|c|c|}
\hline N. $^{\circ}$ & \multicolumn{1}{|c|}{ ACTIVIDAD PRODUCTIVA } & 2016 & $\mathbf{2 0 1 7}$ & 2018 & $\mathbf{2 0 1 9}$ & Total general \\
\hline 1 & $\begin{array}{l}\text { Venta al por menor de prendas de vestir y peletería en } \\
\text { establecimientos especializados. }\end{array}$ & 1062 & 1339 & 1590 & 836 & 4827 \\
\hline 2 & $\begin{array}{l}\text { Venta al por menor de gran variedad de productos en tiendas, } \\
\text { entre los que predominan, los productos alimenticios, las } \\
\text { bebidas o el tabaco, como productos de primera necesidad y } \\
\text { varios otros tipos de productos, como prendas de vestir, } \\
\text { muebles, aparatos, artículos de ferretería, cosméticos, } \\
\text { etcétera. }\end{array}$ & 1009 & 1285 & 1578 & 805 & 4677 \\
\hline 3 & Cultivo de maíz. & 834 & 1449 & 1603 & 477 & 4363 \\
\hline 4 & $\begin{array}{l}\text { Restaurantes, cevicherías, picanterías, cafeterías, etcétera, } \\
\text { incluido comida para llevar. }\end{array}$ & 876 & 1028 & 1205 & 641 & 3750 \\
\hline 5 & $\begin{array}{l}\text { Cría y reproducción de ganado bovino incluido la obtención } \\
\text { de pelo y excremento. }\end{array}$ & 701 & 996 & 1393 & 658 & 3748 \\
\hline 6 & $\begin{array}{l}\text { Actividades de limpiabotas (betuneros), porteadores de } \\
\text { maletas, personas encargadas de estacionar vehículos, } \\
\text { etcétera. }\end{array}$ & 281 & 562 & 1144 & 1199 & 3186 \\
\hline 7 & Cría y reproducción de cerdos. & 384 & 641 & 1122 & 590 & 2737 \\
\hline 8 & Otras actividades profesionales, científicas y técnicas n.c.p. & 707 & 713 & 692 & 485 & 2597 \\
\hline 9 & $\begin{array}{l}\text { Venta al por menor por comisionistas (no dependientes de } \\
\text { comercios); incluye actividades de casas de subastas (al por } \\
\text { menor). }\end{array}$ & 480 & 599 & 785 & 582 & 2446 \\
\hline 10 & Cultivo de cacao. & 495 & 661 & 707 & 371 & 2234 \\
\hline 145 & $\begin{array}{l}\text { Servicios de alojamiento prestados por hoteles, hoteles de } \\
\text { suites, apart hoteles, complejos turísticos, hosterías. }\end{array}$ & 35 & 25 & 30 & 17 & 107 \\
\hline
\end{tabular}

Fuente: Servicio de Rentas Internas del Ecuador (SRI).

Elaborado por: Investigadores.
En contraste, la estadística presentada en la tabla 7 , realizada a partir de la base de datos del Servicio de Rentas Internas (SRI), muestra como año a año se suspenden definitivamente un número considerable de negocios en diferentes actividades productivas, lo cual es preocupante para el crecimiento económico de Crucita.

Tabla 8. Actividades productivas suspendidas por año.

\begin{tabular}{|c|c|c|c|c|c|c|}
\hline & ACTIVIDAD PRODUCTIVA & 2016 & 2017 & 2018 & 2019 & \begin{tabular}{|l} 
Total \\
general
\end{tabular} \\
\hline 1 & $\begin{array}{l}\text { Venta al por menor de gran variedad de productos en tiendas, } \\
\text { entre los que predominan, los productos alimenticios, las } \\
\text { bebidas o el tabaco, como productos de primera necesidad y } \\
\text { varios otros tipos de productos, como prendas de vestir, } \\
\text { muebles, aparatos, artículos de ferretería, cosméticos, } \\
\text { etcétera. }\end{array}$ & 460 & 649 & 4868 & 2492 & 8469 \\
\hline 2 & \begin{tabular}{|l|l} 
Cultivo de maíz. \\
\end{tabular} & 428 & 381 & 4119 & 3383 & 8311 \\
\hline 3 & $\begin{array}{l}\text { Venta al por menor de prendas de vestir y peletería en } \\
\text { establecimientos especializados. }\end{array}$ & 443 & 511 & 4423 & 1772 & 7149 \\
\hline 4 & $\begin{array}{l}\text { Cría y reproducción de ganado bovino incluido la obtención de } \\
\text { pelo y excremento. }\end{array}$ & 332 & 423 & 3985 & 2308 & 7048 \\
\hline 5 & $\begin{array}{l}\text { Restaurantes, cevicherías, picanterías, cafeterías, etcétera, } \\
\text { incluido comida para llevar. }\end{array}$ & 440 & 604 & 3102 & 1458 & 5604 \\
\hline 6 & Cría y reproducción de cerdos. & 179 & 225 & 2595 & 1385 & 4384 \\
\hline 7 & $\begin{array}{l}\text { Venta al por menor de pescado, crustáceos, moluscos y } \\
\text { productos de la pesca en establecimientos especializados. }\end{array}$ & 207 & 229 & 2132 & 899 & 3467 \\
\hline 8 & Cultivo de cacao. & 198 & 265 & 1896 & 980 & 3339 \\
\hline 9 & $\begin{array}{l}\text { Construcción de todo tipo de edificios residenciales: casas } \\
\text { familiares individuales, edificios multifamiliares, incluso } \\
\text { edificios de alturas elevadas, viviendas para ancianatos, casas } \\
\text { para beneficencia, orfanatos, cárceles, cuarteles, conventos, } \\
\text { casas religiosas. incluye remodelación, renovación o } \\
\text { rehabilitación de estructuras existentes. }\end{array}$ & 232 & 306 & 1810 & 665 & 3013 \\
\hline 10 & Otras actividades profesionales, científicas y técnicas n.c.p. & 439 & 565 & 1024 & 827 & 2855 \\
\hline 122 & $\begin{array}{l}\text { Servicios de alojamiento prestados por hoteles, hoteles de } \\
\text { suites, apartamentos hoteles, complejos turísticos, hosterias. }\end{array}$ & 47 & 38 & 68 & 46 & 199 \\
\hline
\end{tabular}

Fuente: Servicio de Rentas Internas del Ecuador (SRI).

Elaborado por: Investigadores.

La siguiente tabla da una perspectiva más clara a la luz de los resultados, mostrando que a pesar de existir gran iniciativa por parte de la ciudadanía para generar actividades económicas, también existe un número importante de organizaciones que han cesado sus actividades, como los datos del año 
2018, en donde se produce el mayor número de actividades económicas suspendidas en la parroquia.

Tabla 9. Actividades productivas aperturadas y suspendidas por año.

\begin{tabular}{|c|c|c|c|c|c|c|c|c|c|}
\hline \multirow{2}{*}{$\frac{N .^{\circ}}{1}$} & \multirow{2}{*}{$\begin{array}{l}\text { ACTIVIDAD PRODUCTIVA } \\
\text { Venta al por menor de prendas de vestir } \\
\text { y peletería en establecimientos } \\
\text { especializados. }\end{array}$} & \multicolumn{2}{|c|}{2016} & \multicolumn{2}{|c|}{2017} & \multicolumn{2}{|c|}{2018} & \multicolumn{2}{|c|}{2019} \\
\hline & & 1062 & 443 & 1339 & 511 & 1590 & 4423 & 836 & 1772 \\
\hline 2 & $\begin{array}{l}\text { Venta al por menor de gran variedad de } \\
\text { productos en tiendas, entre los que } \\
\text { predominan, los productos alimenticios, } \\
\text { las bebidas o el tabaco, como productos } \\
\text { de primera necesidad y varios otros tipos } \\
\text { de productos, como prendas de vestir, } \\
\text { muebles, aparatos, artículos de } \\
\text { ferretería, cosméticos, etcétera. }\end{array}$ & 1009 & 460 & 1285 & 649 & 1578 & 4868 & 805 & 2492 \\
\hline 3 & Cultivo de maíz. & 834 & 428 & 1449 & 381 & 1603 & 4119 & 477 & 3383 \\
\hline 4 & $\begin{array}{l}\text { Restaurantes, cevicherías, picanterías, } \\
\text { cafeterías, etcétera, incluido comida para } \\
\text { llevar. }\end{array}$ & 876 & 440 & 1028 & 604 & 1205 & 3102 & 641 & 1458 \\
\hline 5 & Cría y reproducción de cerdos. & 384 & 179 & 641 & 225 & 1122 & 2595 & 590 & 1385 \\
\hline 6 & $\begin{array}{l}\text { Otras actividades profesionales, } \\
\text { científicas y técnicas n.c.p. }\end{array}$ & 707 & 439 & 713 & 565 & 692 & 1024 & 485 & 827 \\
\hline 7 & Cultivo de cacao. & 495 & 198 & 661 & 265 & 707 & 1896 & 371 & 980 \\
\hline $\begin{array}{c}14 \\
5\end{array}$ & $\begin{array}{l}\text { Servicios de alojamiento prestados por } \\
\text { hoteles, hoteles de suites, apartamento } \\
\text { hoteles, complejos turísticos, hosterías. }\end{array}$ & 35 & 47 & 25 & 38 & 30 & 68 & 17 & 46 \\
\hline
\end{tabular}

Fuente: Servicio de Rentas Internas del Ecuador (SRI).

Elaborado por: Investigadores.

Si bien los desastres desaceleran el crecimiento económico, en términos macroeconómicos, la eficacia y eficiencia de las medidas que se tomen y la predicción de los resultandos esperados dependerá del modelo de análisis macroeconómico que se adopten, como lo menciona Santa Cruz J., (2015) “Un característico del turismo en crucita es su efecto multiplicador donde es esta forma, un cambio en el nivel de la demanda en un sector de la economía no solo afecta a la industria o sector que produce el producto o servicio final, sino también a otras empresas o sectores de la economía" (P.p.54), de ahí que se vuelve sustancial el análisis de las potencialidades de la zona para tomar decisiones que apunten a crear opciones de trabajo más sostenibles y con mayores beneficios sociales para Crucita, dando un espacio para la generación de escenarios en los que el turismo pueda abrirse paso con mayor fuerza.

\section{Conclusiones}

Las bases de datos analizadas tanto del Ministerio de Turismo como del GAD de Portoviejo muestran información que no se corresponde entre sí, lo cual deja una gran brecha del entorno económico del destino turístico de Crucita, a pesar de que la categorización de los sectores es similar, lo cual podría obedecer a la metodología y forma de recojo de información adoptada por cada organización.

De acuerdo con los datos reportados anualmente (período 2016-2019), por el SRI, de denota gran interés por la población de Crucita por incursionar en actividades productivas, lo cual se refleja en la apertura anual de nuevos 
emprendimientos, mostrando que a pesar de las adversidades la ciudadanía no se da por vencida y continúa apostando por opciones que incidan en generar oportunidades de trabajo.

La información analizada evidencia que después del terremoto la parroquia Crucita ha tenido dificultades en sus diferentes sectores económicos, y a pesar de no desistir en su intención emprendedora, existe una alta suspensión de negocios reportada, especialmente durante el año 2018, lo cual sirve de base para la realización de nuevos estudios que permitan identificar las razones por las cuales se suspenden los negocios.

\section{Bibliografía}

Botta, 219-239. Milán: Guerini.

Cuervo, Raimundo (1967) El turismo como medio de comunicación humana, México, Departamento de Turismo.

Da Cruz, José, Jorge Próspero Rozé, Fernando Francia y Gabriela Cob. 2003. Ecología social de los desastres.

Damián Moragues Cortada. 2006. TURISMO, CULTURA $Y$
DESARROLLO. (Fragmentos de la publicación del mismo título, en proceso de edición por AECl). Recuperado de: https://www.oei.es/historico/c ultura/turismodmoragues.htm I

Formichella, M. (2014). El concepto del Emprendimiento y su relación con la educación, el empleo y el desarrollo local. Buenos Aires, Argetina: J.I Massigoge.

García Casado, M., \& Palacios Estremera, M. (2003). Desarrollo sostenible y sustentable en Ecuador. Espacio y desarrollo, 91-92.

Hunziker, Walter y Krapf, Kurt (1942). Grundiss der Allgemeire

Fremderverkehrslebre, Zurich, Plygraphicher verlag.

(MINTUR)., M. d. (14 de septiembre de 2015). El turismo será el eje del cambio de la matriz Placanica en Ecuador. Obtenido de http://www.turismo.gob.ec/elturismo-sera-el-eje-delcambio-de-la-matrizproductiva-en-ecuador/

Muñoz, M. (2012). "Reactivación turística de la parroquia crucita mediante la aplicación indicadores de gestión ambiental y su influencia en los estándares de calidad de los hoteles de la zona". Obtenido de PDF: 
http://repositorio.ucsg.edu.ec/ bitstream/3317/334/1/T-

UCSG-PRE-ESP-AETH14.pdf

Nelson García Reinoso; Yamil Doumet Chilán; Rodney Alfonso Alfonso; (2018). Impacto territorial do terremoto ao setor Turístico de cantón Bolívar, Equador

Naciones Unidas para la Coordinación de Asuntos Humanitarios; ECUADOR LLAMAMIENTO PERÍODO: ABRIL a JULIO de 2016 TERREMOTO OCURRIDO EL 16 DE ABRIL DE 2016

OMT. (1999). Codigo etico mundial para el turismo. Obtenido de https://www.unwto.org/ethicsculture-and-socialresponsibilityes/content/codig o-etico-mundial-para-elturismo

Rivadeneira, M. (2014). El turismo será el eje del cambio de la matriz productiva en Ecuador. Obtenido de Ministerio de Turismo:

https://www.turismo.gob.ec/el -turismo-sera-el-eje-delcambio-de-la-matrizproductiva-en-ecuador/

Placanica, Augusto. 1991. "Lvillo specchio del finimondo: usi storiografici alternativi della tematica catastrofica". En Prodigi, paure, ragione: eventi naturali oggi, editado por Giorgio
Sistema Económico Latinoamericano y del Caribe (SELA) (2016). Recuperado de:

http://www.sela.org/es/prensa /servicioinformativo/20160801/si/2254 $1 /$ comercio-fue-el-masafectado-tras-sismo-de-7-8en-ecuador

Santafeturismo. (2017). Santa Fe Turismo. Obtenido de santa fe turismo:

ttp://www.gov.ar/media/docu mentos/Manual\%20para\%20 Microemprendedores\%20Turi sticos.pdf

Santa cruz, J. (2015). Emprendimiento turístico. En J. Santa cruz, Contextualización (págs. 5455). España: Pedregal.

Trejo, D. (2013). "El desarrollo hotelero y la zona playera: diseño de una hostería alternativa en la parroquia de crucita provincia de Manabí". Obtenido de PDF: http://www.dspace.cordillera. edu.ec/bitstream/123456789/ 1206/1/60-TURISM-13-131717987026.pdf

Villalibre Calderón Cristina (2013). Concepto de urgencia, emergencia, catástrofe, y desastre: revisión histórica y bibliográfica. Universidad de Oviedo. Recuperado de https://digibuo.uniovi.es/dspa ce/bitstream/handle/10651/17 
739/TFM\%20cristina.pdf?seq uence $=3 \&$ is Allowed $=y$

Velásquez, L. M. (2017). http://cnnespanol.cnn.com.

Obtenido de http://cnnespanol.cnn.com:

http://cnnespanol.cnn.com/20 17/04/14/el-turismoenecuador-serecuperalentamentedespues-delterremoto/\#0

Weyl, E. (2014). Comercio. En E. Weyl, características (págs. 78-79). Lima: Publisher S.A.

Zilberstein, J. (2017). nivel de negocios. En J. Zilberstein, Emprendimiento turístico (págs. 81-82). Quito: Universidad de Quito Central. 